\title{
Status of muon neutrino cross section measurements with the T2K on-axis detectors
}

\author{
Kento Suzuki ${ }^{* \dagger}$ \\ Kyoto University \\ E-mail: k.suzuki@scphys.kyoto-u.ac.jp
}

The Tokai-to-Kamioka (T2K) experiment is designed to measure neutrino oscillation parameters. It uses an almost pure muon neutrino $\left(v_{\mu}\right)$ beam that originates at J-PARC. INGRID consists of 14 independent modules ( 7 vertical and 7 horizontal modules), each of which is composed of iron plates and scintillator planes. The modules are arranged in a cross-shape with center on beam-axis and measures the $v_{\mu}$ beam direction and intensity. Another detector called the Proton Module sits on-axis between the horizontal and vertical modules, which is a fully-active tracking detector made of layers of plastic scintillator bars. Measurements of various interaction modes of $v_{\mu}$ interactions in a few $\mathrm{GeV}$ region have been performed with these two on-axis detectors. We present the measurement results of the energy dependent inclusive charged-current cross section on iron, charged-current quasi-elastic and coherent pion production cross section on carbon.

16th International Workshop on Neutrino Factories and Future Neutrino Beam Facilities - NUFACT2014, 25 -30 August, 2014

University of Glasgow, United Kingdom

* Speaker.

${ }^{\dagger}$ On behalf of the T2K collaboration 


\section{Introduction}

In 1998, Super-Kamiokande (Super-K) reported the evidence of neutrino oscillation in 1998 [1]. Since then, many experiments have measured oscillations. But the third mixing angle $\theta_{13}$ only had an upper limit until T2K reported indication of $v_{\mu} \rightarrow v_{e}$ appearance oscillation in 2012. This has now been measured precisely with reactor experiments: $\sin ^{2} 2 \theta_{13}=0.089 \pm 0.011$. Due to the unexpectedly large value for $\theta_{13}, C P$-violating phase $\delta_{C P}$ is now measurable through the neutrino appearance mode. The future measurement of $\delta_{C P}$ requires the other oscillation parameters to be measured precisely. In the current accelerator-based experiments, uncertainty in the neutrino interaction gives the largest systematic error in both appearance and disappearance modes. Understanding of the neutrino interaction mechanism is very essential for the future measurement of $\delta_{C P}$

The Tokai-to-Kamioka (T2K) experiment is a long baseline neutrino oscillation experiment in Japan [2]. T2K utilizes an almost pure $v_{\mu}$ beam, which is generated as follows. An intense $30 \mathrm{GeV}$ proton beam produced at J-PARC main ring strikes a graphite target and produces secondary particles. Positively-charged secondary particles (mainly $\pi^{+}$) are focused by three magnetic horns. The $v_{\mu}$ beam is then produced as the decay products of $\pi^{+}$. The beam is measured at the near detector, ND280, which is located $280 \mathrm{~m}$ downstream from the target. After traveling $295 \mathrm{~km}$, neutrinos are detected at a far detector: Super-K. Oscillation parameters are then determined by measuring the beam before and after oscillation at the near and far detectors, respectively. An overview of the T2K beam-line is shown in left in Figure 1. In the T2K experiment, the direction of beam-axis is shifted by 2.5 degree with respect to that of ND280 and SK. On-axis detectors are located on beam-axis at the near site, which measure the direction and intensity of the neutrino beam. The neutrino energy spectra at the near site are shown in the right in Figure 1. As seen in the figure, the energy spectra are different for different off-axis angles. The on-axis peak energy is $\sim 1.5 \mathrm{GeV}$.

\section{T2K on-axis detectors}

T2K has two types of on-axis detectors at the near site: INGRID and the Proton Module. INGRID [3] has 14 independent modules which are composed of iron plates and scintillator planes. The modules are installed at positions in cross shape whose center is on beam-axis. An overview
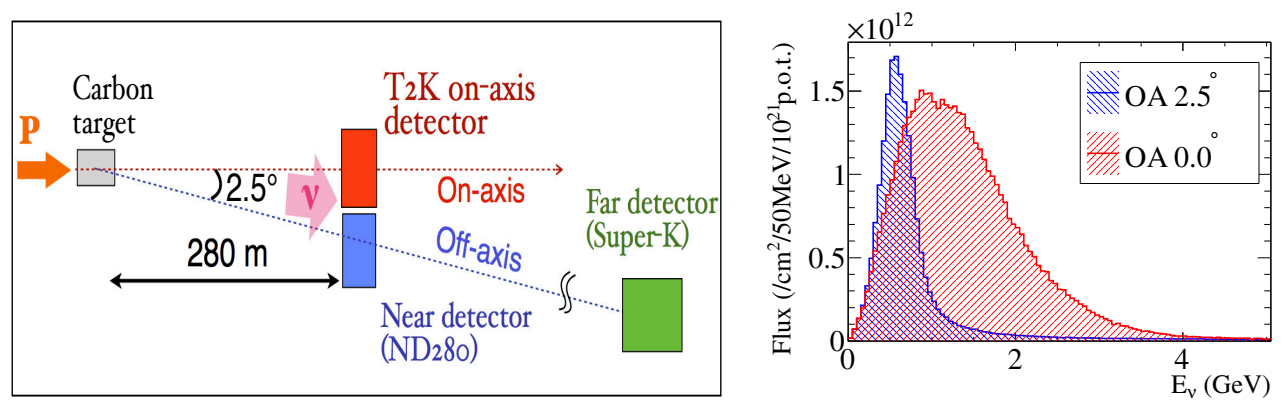

Figure 1: Left: schematic view of the T2K beam-line. The direction of beam-axis is shifted by 2.5 degree with respect to that of ND280 and SK. Right: neutrino energy spectra at the near site. The peak of the energy spectrum is dependent on the off-axis angle. 

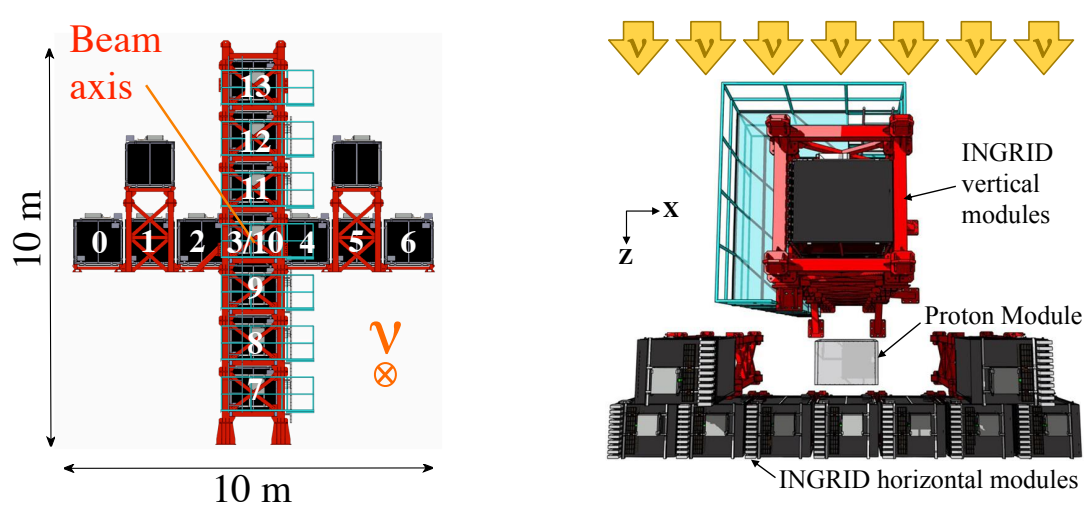

Figure 2: Left: INGRID detector. The modules are arranged to cover $10 \mathrm{~m}$ x $10 \mathrm{~m}$ region to monitor the neutrino beam direction and the event rate stability. The number in each of the modules denotes the module ID. Right: the Proton Module is an extra module located at the beam center between the horizontal and vertical INGRID modules.

of the INGRID detector is shown in left in Figure 2. Each module has 8 iron target plates and 11 plastic scintillator tracking planes. The iron plates and the tracking planes are stacked in alternating layers forming a sandwiched structure. Each of the iron plates has a dimension of $124 \times 124 \mathrm{~cm}^{2}$ and thickness of $6.5 \mathrm{~cm}$, serving a total iron mass of 7.1 ton per module. A scintillator tracking plane consists of two scintillator layers: the layers arranged in the horizontal and vertical directions respectively. Each of the layer has 24 scintillator bars, which lie in a plane of $120 \times 120 \mathrm{~cm}^{2}$ and $1.0 \mathrm{~cm}$ thick along the beam direction. The module is surrounded by VETO planes, which detect charged particles coming from outside of the module. The Proton Module is an extra module located at the beam center between the horizontal and vertical INGRID modules (see right in Figure 2). It consists of 36 tracking layers surrounded by veto planes. Each of the tracking layers contains an array of two types of scintillator bars, making a plane of $120 \times 120 \mathrm{~cm}^{2}$ in the horizontal and vertical directions. In both INGRID and the Proton Module, scintillation light is transmitted by a wavelength shifting (WLS) fiber which is inserted into the scintillator bar. The light is then read out by a Multi-Pixel Photon Counter (MPPC) [4].

\section{Measurement of the energy dependent inclusive charged-current cross section}

Neutrino-iron inclusive charged-current $\left(v_{\mu}\right.$-Fe CC) cross section measurements in the energy range 1-3 GeV are performed with the INGRID detector. The purpose of the measurement is to deteremine the cross sections on iron in the energy range 2-3 GeV which have never been measured.

\subsection{Event selection}

Following event selections are applied to select the neutrino events:

1. Reconstruct tracks of charged particles (mostly $\mu$ ) generated after the neutrino interaction.

2. Veto incoming charged particles such as cosmic muons and sand muons which are generated by neutrino interactions at the upstream wall of INGRID. 

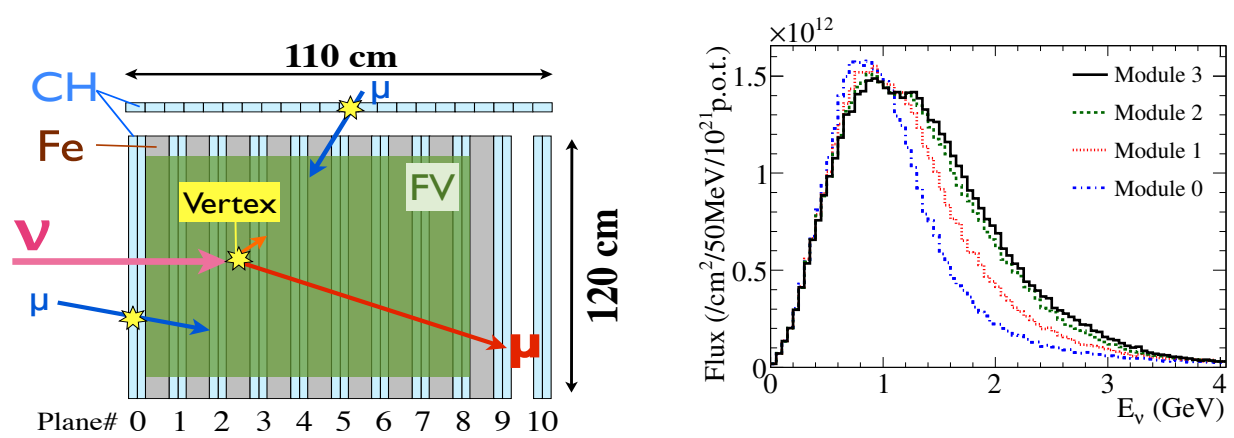

Figure 3: Left: schematic of the neutrino event selection. Right: energy spectra at different modules. Module 0 and 3 are located at the edge and beam-axis in the horizontal direction, respectively.

3. Require the reconstructed vertices to be in a fiducial volume (FV) of the module (see left in Figure 3).

After these selections, the detection efficiencies are estimated with the MC simulation. The CC interactions can be detected with $>70 \%$ efficiency for $E_{V}>1 \mathrm{GeV}$.

\subsection{Analysis method}

Neutrino energy spectra are different at different modules because each of the modules is located at the different off-axis angles (see right in Figure 3). Thus the averaged energy of the neutrino beam is different for the different modules. This feature enables us to extract energy information of the neutrinos. In this analysis, the neutrino events are selected module by module in the way explained in Section 3.1. In addition, the selected events obtained by two modules are merged in order to reduce the effect from a shift of the neutrino beam direction which results in a change of the peak of the neutrino energy spectrum. The definition of the seven module groups is shown in Table 1. The events selected at 7 module groups are used to extract the neutrino energy ${ }^{1}$.

After the selected events are categorized according to different module groups, those events are furthermore categorized according to following two topologies: Downstream (DS-) escaping and NonDS-escaping. If one of the tracks from the neutrino interaction penetrates the most downstream plane, that event is categorized into the DS-escaping. Other than the DS-escaping event, i.e. both of side escaping and fully contained events are categorized into the NonDS-escaping. Events are then categorized according to the vertex position along the beam direction (vertexZ). These event topologies are based on the kinematics of $\mu$ in the final state.

\subsection{Extraction of the neutrino cross section}

The predicted number of CC events is defined as Eq.(3.1):

$$
N_{g j}^{c c}=\sum_{i}\left[\left(1+\Delta f_{i}+\Delta \mathscr{F}_{i g j}^{k}\right) \cdot\left(\phi_{i} \cdot \varepsilon_{i g j} \cdot \sigma_{i}^{c c} \cdot T\right)\right]
$$

\footnotetext{
${ }^{1}$ In this analysis, two separated modules located at off-axis positions are not used.
} 
Table 1: Definition of the module groups. In this analysis, two separated modules located at off-axis positions are not used.

\begin{tabular}{l|ccccccc}
\hline \hline Module group & 1 & 2 & 3 & 4 & 5 & 6 & 7 \\
\hline Module ID & 0,6 & 7,13 & 1,5 & 8,12 & 2,4 & 9,11 & 3,10 \\
\hline Distance from beam-axis (m) & 4.5 & 4.5 & 3.0 & 3.0 & 1.5 & 1.5 & 0.0 \\
\hline \hline
\end{tabular}

Table 2: Systematic error source and the maximum error size on the number of selected events.

\begin{tabular}{c|c|c}
\hline \hline \multicolumn{2}{c|}{ Error source } & Error size (at maximum) \\
\hline \multicolumn{2}{c}{ Flux } & $15 \%$ \\
\hline Detector & uncorrelated error & $2 \%$ \\
\cline { 2 - 3 } & correlated error & $3 \%$ \\
\hline Neutrino interaction & NC interaction & $\begin{array}{c}30 \% \text { for NC } \\
(5 \% \text { for CC+NC) }\end{array}$ \\
\cline { 2 - 3 } & CC interaction & $3 \%$ \\
\cline { 2 - 3 }
\end{tabular}

where indice of $i, g$, and $j$ denote the $i^{\text {th }}$ neutrino energy bin, the $g^{\text {th }}$ module group, and the $j^{\text {th }}$ event topology bin, respectively; $\phi, \varepsilon, \sigma$, and $T$ are the neutrino flux, detection efficiency, CC inclusive cross section predicted by NEUT [5], and the number of target nucleons of iron, respectively; $\Delta \mathscr{F}^{k}$ is the nuisance parameter for $k^{\text {th }}$ systematic error source; $\Delta f$ is a fractional deviation of the CC inclusive cross section. The expected number of events including BG events $\left(=N^{b g}\right)$ is calculated as follows:

$$
N_{g j}^{e x p}=N_{g j}^{c c}+N_{g j}^{b g} .
$$

The BG includes neutral-current (NC) interactions and non- $v_{\mu}$ beam components $\left(\bar{v}_{\mu}\right.$ and $\left.v_{e}\right)$ which are about $15 \%$ and $6 \%$ contributions to the total events, respectively. $N^{\exp }$ is then compared to the number of observed events $\left(=N^{o b s}\right)$ and $\Delta f$, i.e. $\mathrm{CC}$ inclusive cross section, is determined by the least $\chi^{2}$ method:

$$
\chi^{2}=\sum_{g} \sum_{j} \frac{\left(N_{g j}^{o b s}-N_{g j}^{e x p}\right.}{\left(\sqrt{\sum_{k^{\prime}} \sigma_{N_{g j}^{k^{\prime}}}^{2}}\right)^{2}}+\sum_{k}\left(\Delta \overrightarrow{\mathscr{F}}^{k}\right)^{t}\left(V_{k}\right)^{-1} \Delta \overrightarrow{\mathscr{F}}^{k}
$$

where $\sigma_{N_{g j}^{k^{\prime}}}$ is an uncorrelated error for $k^{\prime \text { th }}$ error source including the statistical error for $g^{\text {th }}$ module group and $j^{\text {th }}$ event topology. $V_{k}$ is a covariance matrix for $k^{\text {th }}$ error source. After $\Delta f$ is determined by minimizing $\chi^{2}$ in Eq.(3.3), the $\mathrm{CC}$ inclusive cross sections in the energy range $1-3 \mathrm{GeV}$ are obtained.

Systematic errors on the number of selected events are implemented as covariance matrices in Eq. 3.3. The errors include uncertainties in the neutrino flux, detector response, and cross section. The maximum error size for each error source is listed in Table 2. In addition to the errors, uncertainty in re-interactions of pions which generated after the neutrino interaction is evaluated by comparing hadronic interaction models in GEANT4. The number of neutrino events in each of the models are estimated and cross section fits are done. The difference in the fitting results between the MC samples is then directly taken as the systematics which gives 3-10\% errors on the final results. 


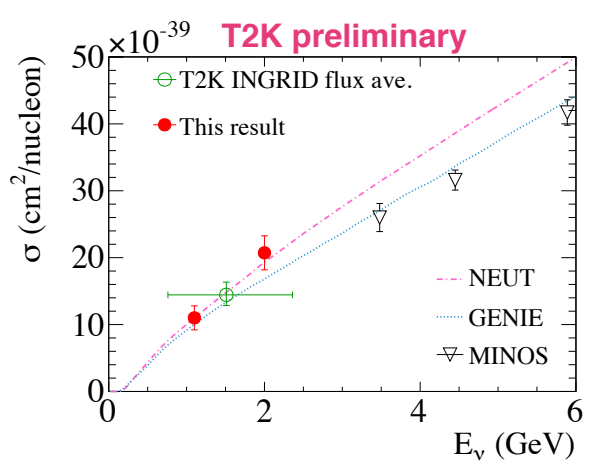

Figure 4: Results of the $v_{\mu} \mathrm{CC}$ inclusive cross section on Fe; $\sigma^{c c}$. Results of other $v_{\mu}$-Fe CC inclusive cross section measurements $[6,7]$ and the prediction by models (NEUT and GENIE [8]) are also shown.

\subsection{Result}

The CC inclusive cross sections on iron at 1.1 and $2.0 \mathrm{GeV}$ are:

$$
\begin{aligned}
& \sigma^{c c}(1.1 \mathrm{GeV})=1.17 \pm 0.18\left(10^{-38} \mathrm{~cm}^{2} / \text { nucleon }\right), \\
& \sigma^{c c}(2.0 \mathrm{GeV})=2.08 \pm 0.24\left(10^{-38} \mathrm{~cm}^{2} / \text { nucleon }\right),
\end{aligned}
$$

with data for $6.27 \times 10^{20}$ protons on target (p.o.t.). These results are shown in Figure 4 with the past measurements $[6,7]$ and predictions by models (NEUT and GENIE [8]). Our measurements are consistent with these predictions.

\section{Measurement of the $\mathrm{CCQE}$ and $\mathrm{CC}$ coherent pion production cross section on carbon}

The measurements of the CC quasi-elastic (CCQE: $v_{\mu}+n \rightarrow \mu+p$ ) and CC coherent pion production $\left(v_{\mu}+A \rightarrow \mu+\pi+A^{\prime}\right)$ cross sections on carbon are performed with the Proton Module and the INGRID horizontal module (hereafter standard module) which is located just downstream of the Proton Module. In both of the measurements, the Proton Module is used as a carbon target and the standard module is used to identify outgoing muons.

\subsection{Event selection}

In both of the analyses, the vertex of the neutrino interaction is required inside the Proton Module. It is then required that there is at least one outgoing $\mu$ track entering into the standard module to select the CC events.

In the CCQE cross section measurement, the CC events are then separated into one- (only $\mu$ ) and two-track ( $\mu$ +proton) samples. For both of the samples, PID is applied based on $d E / d x$ information. For two-track samples, tracks with an opening angle higher than $60^{\circ}$ is selected to enhance the purity of the CCQE samples. In addition to that, selected events are required to have $>150^{\circ}$ of coplanarity angle between the reconstructed tracks. In both one- and two-track samples, events are classified according to topologies of the outgoing $\mu \mathrm{s}$ : if the reconstructed $\mu$ track penetrates the standard module, the event is classified as "high energy sample" while "low 
Table 3: Summary of the systematic errors on the CCQE and CC coherent pion production cross section.

\begin{tabular}{|c|c|c|c|c|c|}
\hline \multirow[t]{2}{*}{ Error souce } & \multicolumn{2}{|c|}{ בCCQE high energy sample } & \multicolumn{2}{|c|}{ "CCQE low energy sample } & \multirow[t]{2}{*}{ CC coherent } \\
\hline & One-track & Two-track & One-track & Two-track & \\
\hline Neutrino flux & $\begin{array}{l}+13.6 \% \\
-11.0 \%\end{array}$ & $\begin{array}{l}+13.9 \% \\
-11.3 \%\end{array}$ & $\begin{array}{l}+17.1 \% \\
-13.6 \%\end{array}$ & $\begin{array}{l}+17.6 \% \\
-14.1 \%\end{array}$ & $\begin{array}{l}+43.2 \% \\
-35.7 \%\end{array}$ \\
\hline Detector response & $4.0 \%$ & $4.7 \%$ & $4.0 \%$ & $6.7 \%$ & $30.9 \%$ \\
\hline Neutrino interaction & $\begin{array}{l}+7.4 \% \\
-5.6 \%\end{array}$ & $\begin{array}{l}+8.5 \% \\
-7.0 \%\end{array}$ & $\begin{array}{l}+10.2 \% \\
-9.5 \%\end{array}$ & $\begin{array}{l}+15.2 \% \\
-10.6 \%\end{array}$ & $\begin{array}{l}+43.2 \% \\
-46.0 \%\end{array}$ \\
\hline Total & $\begin{array}{l}+16.0 \% \\
-13.0 \%\end{array}$ & $\begin{array}{l}+17.0 \% \\
-14.0 \%\end{array}$ & $\begin{array}{l}+20.4 \% \\
-17.0 \% \\
\end{array}$ & $\begin{array}{l}1.0 \% \\
+24.2 \% \\
-18.9 \%\end{array}$ & $\begin{array}{l}+68.5 \% \\
-65.9 \% \\
\end{array}$ \\
\hline
\end{tabular}

energy sample" is classified when the $\mu$ track stopped inside the standard module. The efficiency and purity of these selections are $\sim 80 \%$ and $\sim 30 \%$, respectively. The dominant background is a $\mathrm{CC}$ resonant pion production $(\mathrm{CC} 1 \pi)$, which gives $12-18 \%$ contribution to the $\mathrm{CCQE}$ samples.

For the measurement of the $\mathrm{CC}$ coherent production cross section, two-track ( $\mu$ and $\pi$ ) samples are selected. PID is then applied based on $d E / d x$ information. To discriminate the coherent pion production with CCQE or $\mathrm{CC} 1 \pi$, a kinematic cut is then applied. In this cut, we select $\mu$ 's with a scattering angle less than $15^{\circ}$. The very low momentum transferred for coherent pion production implies small nuclear effects and vertex activity. Therefore events with large activity around the vertex are rejected. The efficiency and purity of this selection are $53 \%$ and $16 \%$, respectively. The dominant background is $\mathrm{CC} 1 \pi$, which gives $24.4 \%$ contribution to the selected samples.

\subsection{Analysis method}

In both CCQE and CC coherent production cross section analyses, the cross section is determined by subtracting BG events, which is simulated using NEUT, from the selected events with the efficiency correction:

$$
\sigma_{C C Q E(C C c o h)}=\frac{N_{C C Q E(C C c o h)}-N_{B G}}{\phi T \varepsilon_{C C Q E}(C C c o h)},
$$

where $N_{C C Q E(C C c o h)}$ and $N_{B G}$ are the number of selected CCQE (CC coherent pion production) and BG events, respectively; $\phi$ is an integrated neutrino flux at the Proton Module; $T$ is the number of target nucleons; $\varepsilon_{C C Q E(C C c o h)}$ is the detection efficiency for the CCQE (CC coherent pion production).

The systematic errors are separated into three sources: neutrino flux, neutrino interaction model and detector response. Table 3 summarizes the error size for each error source.

\subsection{Results}

The CCQE cross sections for high (low) energy samples have a mean neutrino energy of 1.936 $\mathrm{GeV}(0.927 \mathrm{GeV})$ and are measured based on a data sample of $6.04 \times 10^{20}$ p.o.t. This is shown in Figure 5. The left plot in the figure shows the result assuming a relativistic Fermi gas (RFG) model which is implemented in our event generator (NEUT) as a default model. We also attempted to extract the cross section by replacing the RFG with a spectral function, which is known to be a better representation of the nuclear model, and the result is shown in the right plot in Figure 5. In both of the cases, our measurements agree with the GENIE and NEUT. However, we observed a difference in the result between one- and two-track samples in the lower energy region. The difference becomes smaller when the SF model is implemented. 

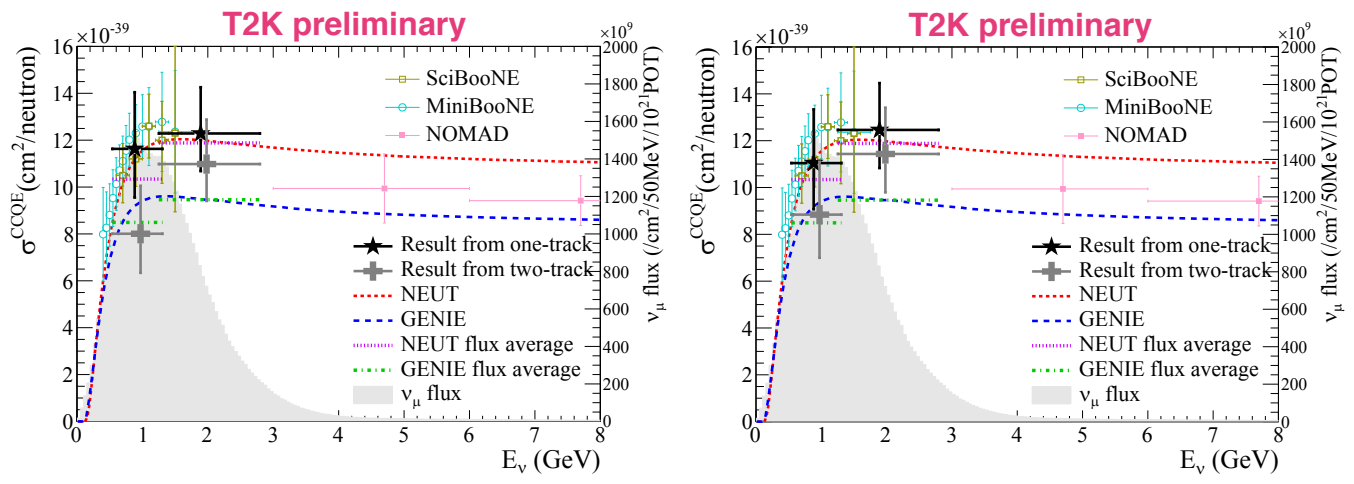

Figure 5: The CCQE cross section result with predictions by NEUT and GENIE. Our data point is placed at the flux mean energy. Left: the result assuming a relativistic Fermi gas model. Right: the result assuming a spectral function model. SciBooNE [9], MiniBooNE [10] and NOMAD [11] results are also plotted.

The $\mathrm{CC}$ coherent pion production cross section at a mean energy of $1.5 \mathrm{GeV}$ is measured using data for $6.04 \times 10^{20}$ p.o.t. We set $90 \%$ C.L. upper limit on the cross section to be $1.98 \times$ $10^{-39} \mathrm{~cm}^{2}$ /nucleus. This result agrees with the prediction by GENIE $\left(1.37 \times 10^{-39} \mathrm{~cm}^{2} /\right.$ nucleus $)$ but is significantly smaller than that by NEUT $\left(3.22 \times 10^{-39} \mathrm{~cm}^{2} /\right.$ nucleus $)$.

\section{Conclusion}

We have performed the measurement of $v_{\mu} \mathrm{CC}$ cross section on iron and carbon in the energy range of 1-3 GeV with the INGRID and Proton Module. We will collect more data to achieve our final target p.o.t. $\left(7.8 \times 10^{21}\right.$ p.o.t. $)$ where the uncertainty in the neutrino interactions could be a limiting factor to obtain precise neutrino oscillation parameters. Therefore, these cross section results are very important for future determination of the fundamental neutrino parameters.

\section{References}

[1] Y. Fukuda et al. (SK Collaboration), Phys. Rev. Lett. 81, 1562 (1998).

[2] K. Abe et al. (T2K Collaboration), Nucl. Instrum. Meth. A659, 106-135 (2011).

[3] K. Abe et al. (T2K Collaboration), Nucl. Instrum. Meth. A 694, 211-223 (2012).

[4] M. Yokoyama et al., Nucl. Instrum. Meth. A 622, 567-573 (2010).

[5] Y. Hayato, Acta Phys. Polon B40, 2477-2489 (2009).

[6] P. Adamson et al. (MINOS Collaboration), Phys. Rev. D 81, 072002 (2010).

[7] K. Abe et al. (T2K Collaboration), Phys. Rev. D 90, 052010 (2014).

[8] C. Andreopoulos et al., Nucl. Instrum. Meth. A614, 87-104 (2010).

[9] Jose Luis Alcaraz-Aunion and Joseph Walding (SciBooNE collaboration), AIP 5 Conf.Proc. 1189,145-150, (2009).

[10] A. A. Aguilar-Arevalo et al., Phys. Rev. D 81, 092005 (2010).

[11] V. Lyubushkin et al. (NOMAD Collaboration), Eur. Phys. J. C63, 355-381 (2009). 